\title{
Xuesaitong injection protects against ischemic stroke by inhibiting the polarization of microglia to M1 phenotype
}

\author{
Fangfang Li, Haiping Zhao, Zhibin Fan, Rongliang Wang, Zhen Tao, Guangwen Li, Yumin Luo \\ Neurology, Xuanwu Hospital of Capital Medical University, China
}

Background: Xuesaitong injection is mainly consisted of panax notoginseng saponins and has shown promise in experimental stroke models, but its effect on long-term recovery after stroke and the related mechanism are unknow. The objective of this study is to investigate the long-term protective effect of Xuesaitong injection against ischemic stroke and its effect on microglia polarization.

Methods: C57BL/6 mice were subjected to middle cerebral artery occlusion (MCAO) for 45 minutes, followed by reperfusion. C57BL/6 mice were randomly divided into five groups, including the sham-operated, vehicle-treated, 15\&mug/g Xuesaitong-treated (LXST), 30\&mug/g Xuesaitong-treated (MXST), 60\&mug/g Xuesaitong-treated (HXST) groups. The animals were immediately injected with Xuesaitong injection through caudal vein at the onset of cerebral reperfusion. 2,3,5-triphenyltetrazolium chloride (TTC) staining was used to assess infract and edema volume, behaviour test was used to evaluate neurological deficits, reverse-transcriptase polymerase chain reaction (PT-PCR) for M1 markers was performed to characterize phenotypic changes in microglia.

Results: TTC staining revealed that Xuesaitong treatment significantly reduced cerebral infract volume and brain edema 24 hours and 3 days after MCAO at all doses compared to vehicle group, accompanied with improved neurological outcomes at 1, 3, 7, 14 days in LXST and MXST groups. PT-PCR measurements revealed a reduction of CD16 (M1 marker) in HXST group 7 days after stroke, as well as a decrease of CD16 and iNOS (M1 markers) in LXST and MXST groups 14 days after stroke.

Conclusions: We demonstrate that low dose of Xuesaitong injection has a long-term neural protective effect against ischemic stroke, possibly by inhibiting the polarization of microglia to M1 phenotype, providing new evidence that Xuesaitong injection might be a promising therapeutic strategy for stroke. 